\title{
Adicción, impulsividad y curvas temporales de deseo
}

\section{Addiction, impulsivity and temporal curves of desire}

\author{
Ginés Juan Cano Cervantes ${ }^{1}$; Francisco Araque \\ Serrano²; Antonio Cándido Ortiz
}

1. Centro Comarcal de Drogodependencias de Linares.

2. Centro Provincial de Drogodependencias de Jaén.

3. Universidad de Granada.

Enviar correspondencia a:

Ginés Juan Cano Cervantes

Centro Comarcal de Drogodependencias de Linares.

Avenida María Auxiliadora, №1 Bajo, Linares (Jaén). C.P. 23700.

E-mail: ginescano@promojaen.es

\section{RESUMEN}

En este estudio se aborda desde las teorías del aprendizaje, el rol de la impulsividad y el deseo de consumo en pacientes con diagnóstico de dependencia de sustancias en abstinencia, consumo habitual y recaída. Para ello, se presenta un nuevo método de valoración del deseo basado en las "curvas de descuento", en el que los pacientes deben describir como perciben la evolución temporal e intensidad de su deseo. Al mismo tiempo, a través de un breve cuestionario sobre emoción y deseo elaborado por los autores y de la Escala de Impulsividad de Plutchik, se pretende comprobar si las emociones positivas o negativas aumentan, disminuyen o no están implicadas en los cambios de deseo e impulsividad. La percepción de la evolución del deseo de los pacientes pertenecientes a los grupos Recaída y Consumo Habitual es similar y presenta una forma hiperbólica, sin embargo, el grupo Recaída presentaba una mayor impulsividad. Además, se halla relación en pacientes de este grupo Recaída entre incremento del deseo de forma hiperbólica, estados placenteros e impulsividad. La mayor puntuación en impulsividad y la evolución hiperbólica del deseo en pacientes que han sufrido recientemente una recaída muestra la necesidad de tener en cuenta y tratar específicamente estos aspectos como relevantes en terapia psicológica y/o farmacológica.

Palabras clave: Autocontrol, craving, descuento hiperbólico, emoción, impulsividad, recaida. recibido: marzo 2010 aceptado: febrero 2011

\section{ABSTRACT}

This study addresses, from theories of associative learning, the impulsivity and craving in patients with a diagnosis of substance dependence in abstinence, habitual use and relapse. We present a new method for the assessment of desire based on discount curve selfdescription, in which patients describe how they perceive the temporal evolution and intensity of their desire. At the same time, through a brief questionnaire about emotion and desire -developed by the authorsand the Plutchik Impulsivity Scale, we set out to check whether positive or negative emotions increase, decrease, or are uninvolved in changes of desire and impulsivity. Perception of the evolution of desire in patients from the Relapse and Habitual Use groups is similar and presents a hyperbolic curve, but the Relapse group presented greater impulsivity. Moreover, a relationship is found in patients from the Relapse group between the hyperbolic increase of desire, the pleasurable states and impulsivity. The higher scores on impulsivity and the hyperbolic evolution of desire in patients who have recently suffered a relapse indicate the need to consider and specifically address these aspects as relevant to psychotherapy and/or pharmacological therapy.

Keywords: Self-control, craving, hyperbolic discounting, emotion, impulsivity, relapse. 


\section{INTRODUCCIÓN}

$\mathrm{T}$ radicionalmente, las teorías explicativas de las adicciones basadas en procesos y mecanismos de aprendizaje se han dividido entre aquellas que ponen el acento en el reforzamiento positivo - placer contingente y contiguo- que se experimenta tras las respuestas de consumo (teorias del incentivo p.e., Hutcheson, Everitt, Robbins y Dickinson, 2001; Robinson y Berridge, 1993), y las que plantean el reforzamiento negativo -alivio tras el escape/evitación del dolor y/o emoción negativa- que se experimenta tras dichas respuestas (p.e. ver Baker, Piper, McCarthy, Majeskie y Fiore, 2004, para revisión; Kenny, Chen, Kitamura, Markou y Koob, 2006) como mecanismo nuclear explicativo de la adicción. Por otra parte, si se añade el factor de demora temporal en la consecución de las recompensas y los castigos, estos enfoques permitirían analizar el comportamiento adictivo desde la perspectiva más amplia y compleja del autocontrol y la impulsividad (Cano y Cándido, 2002a, 2002b).

Tras la emisión de la respuesta de consumo de una sustancia se producen, de forma inmediata -normalmente en cuestión de segundos o minutos- y con una alta probabilidad, efectos recompensantes; ya sea por el placer que se obtiene, por el malestar que se elimina o se alivia temporalmente (físico o afectivo), o por ambos a la vez. Sin embargo, los daños para el individuo y su entorno se demoran en el tiempo -en relación con la rapidez de la obtención del efecto placentero-. Esta capacidad de gratificación inmediata, segura e intensa, frente a los riesgos probables y demorados de sufrir estimulación aversiva (daños) de diversa consideración hace del consumo de drogas y las adicciones en general un excelente ejemplo del comportamiento humano predicho por los estudios experimentales de psicología del aprendizaje, y en especial los referentes al autocontrol y la impulsividad. (Ariely y Wertenbroch, 2002; Calvete y Estévez, 2009; Domjam, 2003; Monterosso y Ainslie, 1999; Rachlin, 1972; Read, Loewenstein y Kalyanamaran, 1999; Verdejo, López-Torrecillas, Orozco y Pérez, 2002).

Una forma de describir gráficamente el juego entre autocontrol e impulsividad es utilizar las denominadas "curvas de descuento" (Vuchinich y Heather, 2003). En ellas se muestra el desarrollo del descuento o pérdida del valor de la recompensa que realiza el sujeto en función del tiempo que tiene que esperar para obtenerla. El tipo de función que sistemáticamente se encuentra en los estudios sobre autocontrol e impulsividad es una pendiente de forma hiperbólica (ver p.e., Killeen, 2009, para revisión). Esto es, que con la progresiva cercanía en el tiempo de las recompensas estas ganan valor, de forma más pronunciada a medida que se aproximan en el tiempo, terminando con un incremento abrupto en su valor cuando su recepción es inmediata, facilitando las respuestas impulsivas de pérdida de control (Ver Figura 1, curva 7). Por otra parte, si dos recompensas alternativas incrementan ambas su valor a una tasa o proporción constante con el transcurso del tiempo, no hay modificaciones por la preferencia de una hacia la otra, sin embargo, con incrementos hiperbólicos del valor de alguna de ellas o de ambas sí que puede explicarse el cambio de preferencia de una recompensa hacia la otra. Por ejemplo, es frecuente que los pacientes en tratamiento por una adicción expresen su preferencia por "llevar una vida normal" o "estar bien con la familia", pero cuando se acerca la recompensa inmediata del consumo esta conducta alternativa incrementa su valor de forma abrupta, hiperbólica, produciéndose el cambio de preferencia. Dilucidar los procesos y mecanismos implicados en este tipo de funciones hiperbólicas y exponenciales, en gran parte derivadas de modelos económicos del comportamiento humano, ha sido y es un importante reto en la investigación básica y clínica (Bickel y Marsch, 2001; Luhmann, 2009; Vuchinich y Heather, 2003), pues entre otras cuestiones permitiria optimizar las decisiones terapéuticas en función de la curva de descuento temporal del paciente.

Los resultados de las investigaciones muestran que las tasas de descuento hiperbólico de la recompensa -la pendiente de la curva- son mayores en consumidores de drogas que en no consumidores (Kirby y Petry, 2004; Madden, Petry, Badger y Bickel, 1997). Un dato muy interesante es que estas tasas de descuento hiperbólico son más pronunciadas si el sujeto se encuentra bajo los síntomas de abstinencia y "craving" (Field, Santarcangelo, Sumnall, Goudie y Cole, 2006; Giordano, Bickel, Lowenstein, Jacobs, Marsch y Badger, 2002), lo que puede indicar que el valor de la recompensa aumenta debido a la intensidad de los efectos negativos que esta sufriendo y las posibilidades de aliviarlos o eliminarlos mediante el consumo.

El "craving", y su control por parte de la persona que sufre una adicción, es considerado un constructo central a la hora de explicar el desarrollo, mantenimiento, abstinencia y recaída en adicciones (Becoña y Cortés, 2008; Drumond, 2001; Weiss, 2005). El deseo intenso, urgencia, ansia o "craving" ha sido relacionado tanto con el escape/evitación de estados aversivos del síndrome de abstinencia u otros estados físicos o afectivos negativos, como con la búsqueda del incentivo de estados placenteros. Si en la conducta de evitación se estudia el miedo como mecanismo mediador y el alivio como incentivo (Cándido, González y De Brugada, 2004), en el consumo de sustancias puede considerarse el deseo otro de los mecanismos mediadores en las conductas de aproximación y consumición de la sustancia, que facilitan el comportamiento impulsivo que provoca la pérdida de control. El deseo es medido en pacientes, además de por la valoración subjetiva de los informes verbales durante las entrevistas clínicas, a través de medidas unidimensionales, cuestionarios de personalidad, medidas psicofisiológicas, de elección, etc. (Badger, Bickel, Giordano, Jacobs, Lowenstein y Marsch, 2007; Jiménez, Graña, Montes y Rubio, 2009; Llopis y Castillo, 2008; Sayette, Shiffman, Tiffany, Niaura, Martin y Shandel, 2000). Sin embargo, desde nuestro conocimiento, no hay un instrumento de medida que relacione el curso temporal del deseo subjetivo de droga con la impulsividad medida a través de procesos de descuento de la recompensa.

Este estudio exploratorio presentaba tres objetivos: en primer lugar, describir la intensidad y evolución temporal del deseo subjetivo de consumo de sustancias por pacientes adictos a sustancias. Para ello, se presenta un nuevo 
método de valoración subjetiva del "craving" basado en las curvas de descuento, a través del cual valorar la dinámica de los cambios del deseo a lo largo de diferentes intervalos temporales, ya sea entre cortos periodos de consumo durante el mantenimiento del proceso adictivo o entre periodos más amplios durante los procesos de abstinencia y recaída. En segundo lugar, se pretendia ver si existe relación existente entre deseo e impulsividad en pacientes en diferentes procesos terapéuticos: abstinencia, consumo habitual y recaída. Finalmente, se pretendia valorar la influencia que los factores emocionales, positivos o negativos, tienen sobre la intensidad del deseo subjetivo de consumo.

\section{MÉTODO}

\section{Participantes}

Participaron 66 pacientes (10 mujeres y 56 hombres) en tratamiento ambulatorio en el Centro Comarcal de Drogodependencias de Linares, con diagnóstico clínico según criterios DSM-IV de al menos dependencia de una sustancia, resultando: 15 dependientes de alcohol, 18 de cocaína, 18 de heroína + cocaína, 7 de cocaína + alcohol, 5 de heroína, 1 de cánnabis, 1 de tranquilizantes y 1 de morfina.

\section{Procedimiento}

Las diferentes pruebas fueron realizadas en horario de 8 a 15 horas en el Centro Comarcal de Drogodependencias de Linares, en la consulta del psicólogo y previa a la misma. Se les informó que las pruebas que iban a realizar formaban parte de una investigación y dieron su consentimiento.

Los pacientes fueron asignados a tres grupos en función del estado del proceso terapéutico: Recaída, Consumo Habitual y Abstinencia. Los pacientes del grupo Recaída (23 pacientes) son aquellos que han sufrido recientemente una recaída en el consumo de la sustancia, tras al menos un mes de abstinencia (rango de 1 mes a 8 años). Los pacientes del grupo Consumo Habitual (23 pacientes) son aquellos que llevan consumiendo al menos durante un periodo de 6 meses de forma continuada (rango de 20 minutos a 1 semana). Los pacientes del grupo Abstinencia (20 pacientes) son aquellos que llevan al menos 1 mes sin consumir la sustancia (rango de 1 mes a 6 años).

\section{Material}

Se utilizaron diversas pruebas de impulsividad, deseo y estados afectivos. Para medir el grado de conducta impulsiva se les administró a los pacientes la Escala de Impulsividad de Plutchik (Páez, Jiménez, López, Raull, Ortega y Nicolini, 1996; Plutchik y Van Praag, 1989; Rubio, Montero, Jáuregui, Martinez, Álvarez y Marín, 1998). Esta escala es autoaplicada y consta de 15 ítems, todos relacionados con una posible falta de control sobre determinadas conductas. La puntuación total puede oscilar entre 0 y 45 puntos.
El curso temporal del deseo subjetivo entre consumiciones se midió a través de una prueba diseñada por los autores para este estudio y basada en las denominadas "curvas de descuento". En papel tamaño A4 y de forma gráfica se le presentan a los pacientes 8 recuadros. Los pacientes han de señalar, de entre siete recuadros que contienen siete curvas o líneas que indican diferentes formas de cambio de deseo con el paso del tiempo, aquella que mejor se ajuste a los cambios en su deseo de consumo. Un octavo recuadro en blanco puede ser elegido para dibujar ellos la curva o línea, en el caso de que ninguna se ajuste a los cambios en su deseo con el paso del tiempo (ver Figura 1). Los pacientes del grupo Recaída tenían que señalar 0 dibujar la curva o línea que indicara el cambio en el deseo, en el periodo comprendido desde que dejaron de consumir hasta que se produjo la recaída. Los pacientes del grupo Consumo Habitual tenían que señalar o dibujar la curva o línea que indicara el cambio en el deseo entre episodios de consumo. Los pacientes del grupo Abstinencia tenían que señalar o dibujar la curva o línea que indicara el cambio en el deseo, en el periodo comprendido desde que dejaron de consumir hasta la actualidad. En general, los pacientes son capaces de comprender la tarea. Aquellos que tienen dificultad de lectura o comprensión de la tarea se les lee o se les da alguna explicación adicional. Por ejemplo "El deseo puede aumentar, disminuir o mantenerse con el paso del tiempo. En la siguiente página tienes siete recuadros con siete líneas o curvas que indican que el deseo aumenta, disminuye 0 se mantiene con el paso del tiempo. Señala la que mejor se ajuste al cambio en tu deseo". Tres de los pacientes no realizaron las pruebas debido al estado de intoxicación que presentaban. Se supervisaba la realización de las pruebas al finalizar las mismas ya que a veces dejaban alguna prueba 0 pregunta sin contestar.

Finalmente, se les administró un corto cuestionario sobre la relación subjetiva entre deseo y emoción. A los pacientes del grupo Abstinencia se les pregunta si sentirse bien 0 sentirse mal les produce aumento del deseo de consumo. A los pacientes del grupo de Consumo Habitual se les pregunta si el consumo de la sustancia es para producirle una emoción positiva sintiéndose bien, aliviar una emoción negativa o para evitarla. A los pacientes del grupo Recaída, qué tipo de emoción sentían (positiva o negativa) previa a la recaída y qué tipo de emoción buscaban conseguir. Estas preguntas fueron agrupadas en tres categorias en orden a igualar a los distintos grupos: deseo si bien, deseo si mal y deseo evitación. Los pacientes del grupo Abstinencia no respondieron a preguntas que se encuadran en la última categoría.

\section{RESULTADOS}

Curvas temporales de deseo. La Tabla 1 refleja el número y porcentaje de pacientes en cada grupo que eligen cada curva o línea de deseo. Como se puede observar en los datos de la Tabla 1, los grupos de Recaída y Consumo habitual perciben una evolución del deseo de forma hiperbólica: curva 7 (hiperbólica estable) y 2 (hiperbólica inestable) y 

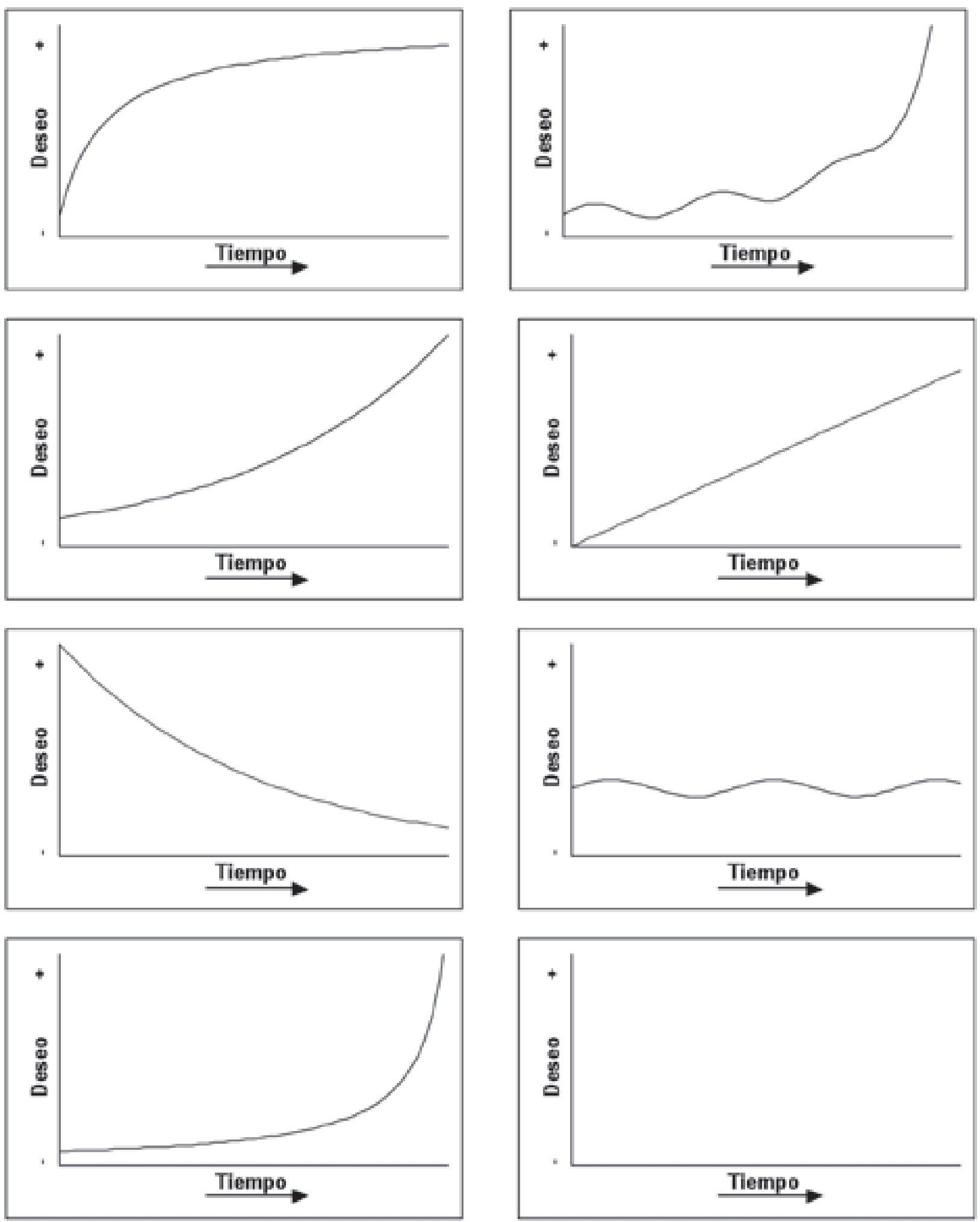

Figura 1. Curvas de deseo. Evolución del deseo en función del tiempo. De izquierda a derecha y de arriba abajo: curvas 1, curva 2 (hiperbólica inestable), curva 3 (exponencial), línea 4 (incremento lineal), curva 5 (descenso), curva 6 (sostenido e inestable), curva 7 (hiperbólica estable), 8 (recuadro en blanco).

curva 6 (deseo sostenido-inestable). En el grupo Abstinencia la curva más elegida fue la 5 (descenso del deseo). Es interesante hacer notar que en ningún caso fue elegida la línea 4 (incremento lineal del deseo). Esta descripción fue corroborada mediante el análisis estadístico mediante la prueba $\chi^{2}$ (corrección Monte Carlo), que mostró que figura y grupo de adicción estaban relacionadas $\left(\chi_{12,1}^{2}=32,453\right.$; $p<0,001$. Coeficiente de Contingencia $C=0,57)$. 
Tabla 1. Número y porcentaje de pacientes por grupos que eligen cada curva o línea de deseo .

\begin{tabular}{ccccccc}
\hline & \multicolumn{2}{c}{ Gr. Recaída } & \multicolumn{2}{c}{ Gr. Consumo Habitual } & \multicolumn{2}{c}{ Gr. Abstinencia } \\
\hline $\begin{array}{c}\text { Curvas o líneas } \\
\text { de deseo }\end{array}$ & Recuento & $\%$ & Recuento & $\%$ & Recuento & $\%$ \\
\hline 1 & 0 & 0,00 & 2 & 8,70 & 0 & 0,00 \\
2 & 5 & 21,70 & 5 & 21,70 & 0 & 0,00 \\
3 & 1 & 4,30 & 0 & 0,00 & 0 & 0,00 \\
5 & 3 & 13,00 & 2 & 8,70 & 13 & 65,00 \\
6 & 6 & 26,10 & 6 & 26,10 & 3 & 15,00 \\
7 & 6 & 26,10 & 6 & 26,10 & 0 & 0,00 \\
8 & 2 & 8,70 & 2 & 8,70 & 4 & 20,00 \\
\hline
\end{tabular}

Escala de Impulsividad de Plutchik. Se realizó un análisis de varianza unifactorial (ANOVA) para medir diferencias en impulsividad entre grupos Recaída, Consumo Habitual y Abstinencia. Como se observa en la Figura 2, los grupos se escalan en su nivel de impulsividad de mayor (Recaida) a menor (Abstinencia).

Las puntuaciones directas se trasformaron a su raíz cuadrada para cumplir el criterio de homogeneidad de varianza (prueba de Levene). Se obtuvieron diferencias estadisticamente significativas entre los grupos $\left(F_{2,63}=\right.$ $6,446 ; p<0,003)$. El análisis de estas diferencias mediante la prueba DMS mostró diferencias entre el grupo de Recaída y Abstinencia $(p<0,001)$, y cercanas a la significatividad estadística entre Recaída y Consumo Habitual $(p=0,06)$, y entre los grupos de Consumo habitual y Abstinencia ( $p=$ $0,07)$. Por otra parte, es de resaltar que el único grupo que supera la puntuación 20, punto de corte que consideran impulsividad patológica los autores en la adaptación española del cuestionario de impulsividad de Plutchik (Rubio et al., 1988), es el de Recaída, con una puntuación media de 22,78 .

Cuestionario emoción y deseo. Cuando se reorganizan los datos de impulsividad para incluir las preguntas dicotómicas del cuestionario (categorías "deseo si bien", "deseo si mal" y "deseo evitación") y se analizan éstos en cada grupo mediante comparaciones utilizando la t de Student, sólo

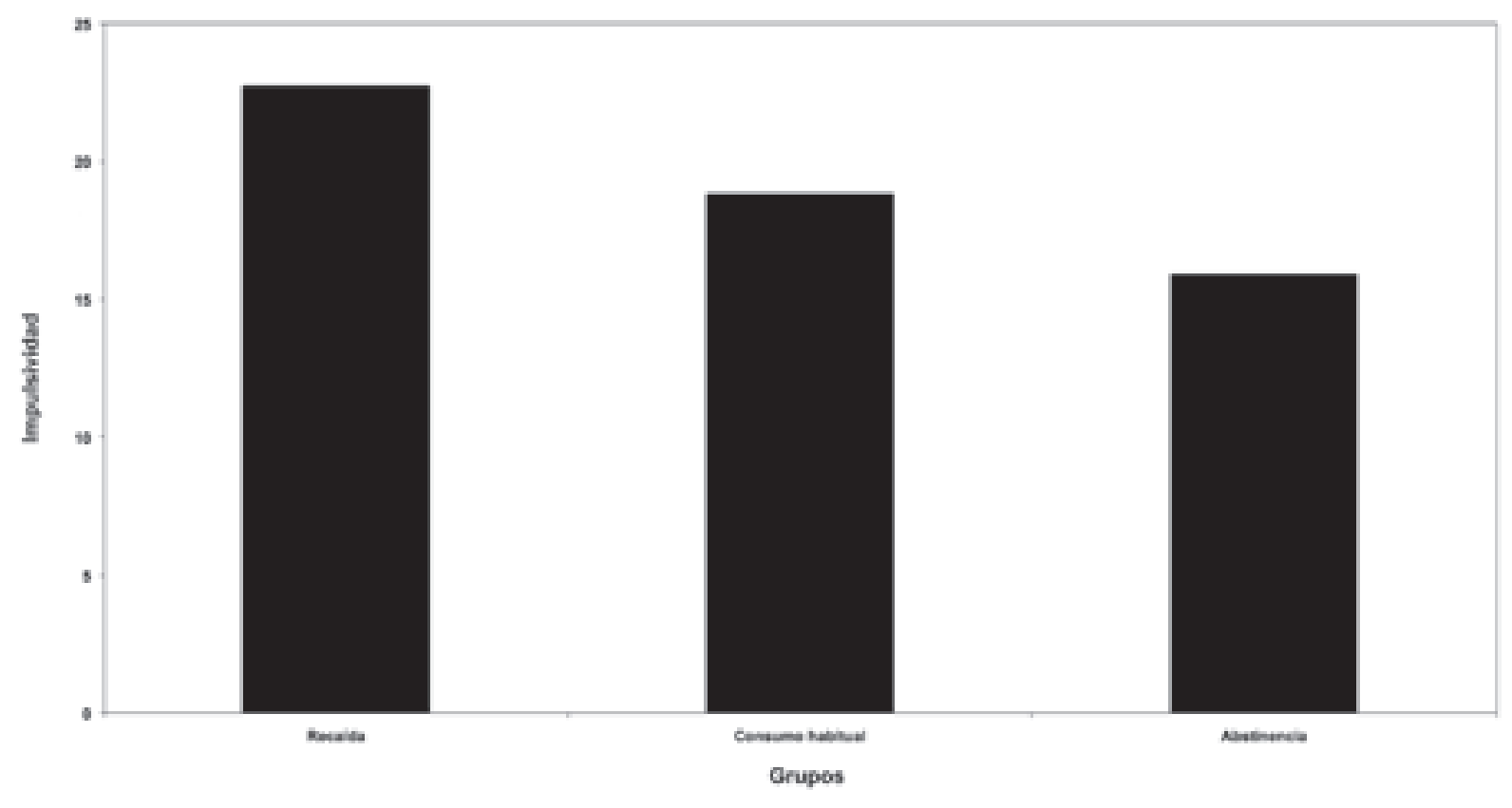

Figura 2. Representación de la puntuación en impulsividad (Escala de Plutchik) de los sujetos por grupos. 
se muestran diferencias estadísticamente significativas en el grupo de Recaída, ante la pregunta de si consumieron encontrándose bien para encontrarse mejor $\left(t_{1,21}=2,429 ; p\right.$ $=0,024$ ), y una mayor impulsividad (una puntuación media de 26,45 , claramente superior al punto de corte) en los pacientes que contestan afirmativamente a esa pregunta.

\section{DISCUSIÓN}

El primer objetivo de este estudio era describir la intensidad y evolución temporal del deseo subjetivo de consumo de sustancias mediante curvas o líneas que describieran la evolución del deseo subjetivo entre consumos.

A este respecto, el primer dato a destacar es la ausencia de incrementos lineales del deseo entre consumos (ver Figura1, línea de deseo 4), de forma que los pacientes no suelen percibir sus cambios de deseo de consumo como incrementos constantes del deseo en función del tiempo. Esto es, como una función lineal. También es mínima la presencia de aumentos del deseo de consumo de forma exponencial (ver Figura1, curva de deseo 3), pues sólo un sujeto describe aumentos de deseo de forma exponencial con el paso del tiempo hasta la recaída. Sin embargo, los aumentos de deseo de forma hiperbólica (ver Figura 1, curvas de deseo 2 y 7) son los más frecuentemente señalados, tanto por los pacientes que recientemente han sufrido una recaída como por los que están consumiendo regularmente; esto concuerda con la literatura de los estudios de elección y decisión basados en la metodología de las "curvas de descuento", donde las elecciones basadas en un patrón hiperbólico del cambio en las preferencias es el resultado más común, especialmente y de manera más acusada en adictos a sustancias (Madden et al., 1997; Kirby y Petry, 2004). Este patrón muestra un deseo intenso o "craving" justo antes del consumo, lo cual se ajusta a la idea que se plantea al inicio de este estudio, al proponer que el deseo intenso "craving" es un factor clave mediador en la pérdida de control que sufren un elevado número de pacientes adictos a sustancias, y que acompaña al cambio de preferencia -desde la abstinencia y hacia la recompensa inmediata del consumo-. Sin embargo, el que un alto porcentaje de pacientes presenten alteraciones en su deseo durante gran parte del intervalo entre consumos (ver Figura 1, curva de deseo 2) nos puede indicar que podrían ser diferentes los mecanismos implicados en la aparición del deseo subjetivo y las estrategias de autocontrol empleadas por el sujeto o, al menos, podría tomarse como un índice de su efectividad en el control del deseo por el consumo. Investigaciones futuras deberían estudiar estos aspectos, utilizando la descripción de las curvas de deseo como otro índice en la elección de la estrategia terapéutica a adoptar. Además, este patrón hiperbólico de incremento del deseo plantea la tesis de que existan similares mecanismos de incremento del deseo entre breves periodos de consumo (en pacientes que están consumiendo regularmente) y entre amplios periodos de consumo (en pacientes que han sufrido una recaida). A este respecto también sería interesante investigar los patrones de incremento del deseo en personas adictas que no acuden a tratamiento.
Por otra parte, un porcentaje significativo de pacientes en los grupos Consumo Habitual y Recaída señalan un deseo constante e inestable (ver Figura1, curva de deseo 6), pero sin un abrupto incremento final previo al consumo y recepción de la recompensa; esto pone de manifiesto que, aunque en todos los pacientes el manejo del deseo es un factor clave a tener en cuenta en terapia, la pérdida de control mediada por el "craving" es más importantes en aquellos pacientes que muestran un patrón hiperbólico de deseo.

Finalmente, que en el grupo de pacientes abstinentes la curva de deseo 5 haya sido la más elegida no hace sino poner de manifiesto que el éxito de la terapia respecto a la ausencia de consumo está inversamente relacionada con el aumento del deseo por la sustancia. Sin embargo, existe un alto porcentaje de pacientes que deciden ellos dibujarla (ver Figura1, recuadro en blanco), quizá debido a que la curva 5 es la única opción a elegir en la que se produce un descenso del deseo con el paso del tiempo. Se hace por ello necesario, para posteriores investigaciones con pacientes abstinentes, ampliar el número de curvas o líneas que muestren descenso en los niveles de deseo con el transcurso del tiempo.

En segundo lugar, se pretendía ver si existe relación entre deseo e impulsividad en pacientes en diferentes procesos terapéuticos: abstinencia, consumo habitual y recaída. El primer dato a destacar son las diferencias en impulsividad entre los tres grupos a estudio (ver Figura 2). Los pacientes que han sufrido recientemente una recaída son los que mayor puntuación obtienen en el cuestionario de impulsividad, por encima del punto de corte, lo que muestra la relevancia de este aspecto clínico a la hora de abordar el tratamiento psicológico o farmacológico de las recaídas. Sin embargo, una comparación más detallada entre los distintos grupos de pacientes, la evolución subjetiva de su deseo de consumo e impulsividad no pudo realizarse de forma estadísticamente razonable debido a lo escaso de la muestra y dispersión de los pacientes. Esta es una limitación importante del estudio que debería tratarse de forma directa en investigaciones futuras. Podría mencionarse, sin embargo, que los pacientes mas impulsivos (media de 26.2) resultaron ser aquellos que en el grupo de Recaída describian la evolución de su deseo mediante la curva 2 (hiperbólica inestable), lo que insistiría en la existencia de una fuerte relación entre impulsividad, pérdida de control, intensidad creciente súbita del deseo ("craving") y recaída.

Finalmente, se pretendia valorar la influencia que los factores emocionales, positivos o negativos, tienen sobre el deseo subjetivo de consumo e impulsividad. A este respecto, hay que mencionar que no existen diferencias entre los que consumen bajo diferentes estados emocionales, excepto en el grupo de Recaída, donde los pacientes que puntúan significativamente más alto en impulsividad afirman que la recaída se produjo sintiéndose bien y con la finalidad de sentirse mejor, estableciéndose una interesante relación entre recaída, búsqueda de incentivo de estados placenteros e impulsividad. Nuestros datos parecen indicar que en el grupo de Recaída, un apreciable porcentaje de sujetos, este ansia por el consumo de droga parece provocarlo la expectativa de sus propiedades recompensantes, su incentivo (Hutcheson 
et al., 2001; Robinson y Berridge, 1993), sobre todo cuando su estado afectivo es positivo; esto puede ser debido a que estos estados afectivos positivos son psicológicamente mas "expansivos" y tienden a aumentar la impulsividad (Youen y Lee, 2003) y, por lo tanto, reducir el autocontrol ante la conducta de consumo.

En este entorno teórico y clínico hemos propuesto un nuevo método de valoración en el que los pacientes adictos describen la percepción de la evolución de su deseo, relacionándola con la pérdida de control y niveles de impulsividad en diferentes fases del proceso terapéutico. Estudios futuros deben insistir en este tipo de relaciones con el fin de planificar nuevas estrategias de evaluación e intervención basadas en el desarrollo de técnicas de autocontrol, en las que el individuo tenga un papel más activo en la identificación y control de las respuestas condicionadas de deseo y la impulsividad, que pueden mediar en la pérdida o disminución del control de su propio comportamiento.

\section{REFERENCIAS}

Ariely, D. y Wertenbroch, K. (2002). Procrastination, deadlines and performance: self-control by precommitment, Psychological Science, 13, 219-224.

Badger, G.J., Bickel, W.K., Giordano, L.A., Jacobs, E.A. Lowenstein, G. y Marsch, L. (2007). Altered states: the impact of immediate craving on the valuation of current and future opioids. Journal of Health Economics, 29, 865-876.

Baker, T.B., Piper, M.E., McCarthy, D.E., Majeskie, M.R. y Fiore, M.C. (2004). Addiction motivation reformulated: an affective processing model of negative reinforcement. Psychological Review, 111, 33-51.

Becoña, E. y Cortés, M. (Eds.) (2008). Guía clínica de intervención psicológica en adicciones. Socidrogalcohol. Barcelona.

Bickel, W.K. y Marsch, L.A. (2001). Toward a behavioral economic understanding of drug dependence: delay discounting processes. Addiction, 96, 73-86.

Calvete, E. y Estevez, E. (2009). Consumo de drogas en adolescentes: el papel del estrés, la impulsividad y los esquemas relacionados con la falta de límites. Adicciones, 21, 49-56.

Cándido, A., González, F. y De Brugada, I. (2004). Safety signals from avoidance learning but not from yoked classical conditioning training pass both summation and retardation tests for inhibition. Behavioural Processes, 66, 153-160.

Cano, G.J. y Cándido, A. (2002a). Análisis psicológico de las estrategias de autocontrol implicadas en los programas de reducción de daños y riesgos del consumo de heroina. Revista Española de Drogodependencias, 27, 52-65.

Cano, G.J. y Cándido, A. (2002b). Análisis psicológico conductual de las estrategias de autocontrol implicadas en el consumo de alcohol. Adicciones, 14, 443-454.

Domjam, M. (2003). Principios de Aprendizaje y de Conducta. Madrid: Thomsom.
Drummond, D.C. (2001). Theories of drug craving, ancient and modern. Addiction, 96, 33-46.

Field, M., Santarcangelo, M., Sumnall, H., Goudie, A. y Cole, J. (2006). Delay discounting and the behavioural economics of cigarette purchases in smokers: The effects of nicotine deprivation. Psychopharmacology, 186, 255-263.

Giordano, L.A., Bickel, W.K., Lowenstein, G., Jacobs, E.A., Marsch, L. y Badger, G.J. (2002). Mild opioid deprivation increases the degree that opioid-dependent outpatients discount delayed heroin and money. Psichopharmacology, 163, 174-182.

Hutcheson, D.M., Everitt, B.J., Robbins, T.W. y Dickinson, A. (2001). The role of withdrawal in heroin addiction: enhances reward or promotes avoidance? Nature Neuroscience, 4, 943-947.

Jimenez, M., Graña, J.L., Montes, V., y Rubio, G. (2009). Alcohol craving scale based on three factors. European Addiction Research, 15, 135-142.

Kenny, P.J., Chen, S.A., Kitamura, O., Markou, A. y Koob, G.F. (2006). Conditioned withdrawal drives heroin consumption and decreases reward sensitivity. Journal of Neuroscience, 26, 5894-5900.

Killen, P.R. (2009). An additive-utility model of delay discounting. Psychological Review, 116, 602-619.

Kirby, K.N. y Petry, N.M. (2004). Heroin and cocaine abusers have higher discount rates for delayed rewards than alcoholics or non drug using controls. Addiction, 99, 461-471.

Luhmann, C.C. (2009). Temporal decision-making: insight from cognitive neuroscience. Frontiers in Behavioral Neuroscience. 3, Article 39. doi:10.3389/neuro.08.039.2009.

Llopis, J.J. y Castillo, A. (2008). Utilidad del tratamiento con oxcarbacepina en pacientes con diagnóstico de trastorno por abuso/dependencia de cocaína. Adicciones, 20, 263-270.

Madden, G.J., Petry, N.M., Badger, G.J. y Bickel W.M. (1997). Impulsive and self-control choices in opioid-dependent patients and nondrug-using control participants: drug and monetary rewards. Experimental and Clinical Psychopharmacology, 5, 256-262.

Monterosso, J. y Ainslie, G. (1999). Beyond discounting: possible experimental models of impulse control. Psychopharmacology, 146, 339-347.

Páez, F., Jiménez, A., López, A., Raull, J.P., Ortega, H. y Nicolini, H. (1996). Estudio de validez de la traducción al castellano de la Escala de Impulsividad de Plutchik. Salud Mental, 19 (sup/ 3), $10-12$.

Plutchik, R. y Van Praag, H.M. (1989). The measurement of suicidality and impulsivity. Progess in Neuro-psychopharmacology and Biological Psychiatry, 13(Supp), 23-24.

Rachlin, H. y Green, L. (1972). Commitment, choice and self-control. Journal of the Experimental Analysis of Behaviour, 17, 15-22.

Read, D., Loewenstein, G. y Kalyanamaran, S. (1999). Mixing virtue and vice: combining the immediacy effect and the diversification heuristic. Journal of Behavioral Decision Making, 12, 257-273. 
Robinson, T.E. y Berridge, K.C. (1993). The neural basis of drug craving: an incentive-sensitization theory of addiction. Brain Research Reviews, 18, 247-291.

Rubio, G., Montero, I., Jáuregui, J., Martinez, M.L., Álvarez, S. y Marín, J.J. (1998). Validación de la escala de impulsividad de Plutchik en población española. Archivos de Neurobiología, 61, 223-232.

Sayette, M.A., Shiffman, S., Tiffany, S.T., Niaura, R.S., Martin, C.S. y Shandel, W.G. (2000). The measurement of drug craving. Addiction, 95, 189-210.
Weiss, F. (2005). Neurobiology of craving, conditioned reward and relapse. Current Opinion in Pharmacology, 5, 9-19.

Verdejo, A., López-Torrecillas., Orozco, C. y Pérez, M. (2002). Impacto de los deterioros neuropsicológicos asociados al consumo de sustancias sobre la práctica clínica con drogodependientes. Adicciones, 14, 1-26.

Vuchinich, R.E. y Heather, N. (2003). Choice, behavioural economics and addiction. Oxford: Pergamon.

Youen, K.S.L. y Lee, T.M.C. (2003). Could mood state affect risktaking decisions? Journal of Affective Disorders, 75, 11-18. 